\title{
MINIMAL SUPERADDITIVE EXTENSIONS OF SUPERADDITIVE FUNCTIONS
}

\author{
ANDREW BRUCKNER
}

Introduction. A real valued function $f$ is said to be superadditive on an inverval $I=[0, a]$ if it satisfies the inequality $f(x+y) \geqq$ $f(x)+f(y)$ whenever $x, y$ and $x+y$ are in $I$. Such functions have been studied in detail by E. Hille and R. Phillips [1] and R. A. Rosenbaum [2]. In this paper we show that any superadditive function $f$ on $I$ has a minimal superadditive extension $F$ to the non-negative real line $E$, and then proceed to show that $F$ inherits much of its behavior from the behavior of $f$. We deal primarily with superadditive functions which are continuous and non-negative.

A simple example of a superadditive function on $[0, a]$ is furnished by a convex function $f$ with $f(0) \leqq 0$. Also, if $f$ is convex and $f(0)=0$, then it is easy to verify that its minimal superadditive extension $F$ is given by

$$
F(x)=n f(a)+f(x-n a)
$$

for $n a \leqq x<(n+1) a$. In general, the minimal superadditive extension $F$ is not easily computed. In the sequel we shall discuss two methods for obtaining $F$. For brevity we shall use the notation $f^{*} F$ to mean " $F$ is the minimal superadditive extension of $f$ ".

1. The decomposition method. Definition. Let $x \in E$. The numbers $x^{1}, \cdots, x^{n}$ are said to form an $a$-partition for $x$ if $x^{1}+\cdots+x^{n}=x$ and for each $i=1, \cdots, n$ we have $0 \leqq x^{i} \leqq a$.

THEOREM 1. Let $f$ be a superadditive function on $I=[0, a]$. Then the function $F$ defined on $E$ by the equation

$$
F(x)=\sup \Sigma f\left(u^{i}\right),
$$

the supremum being taken over all a-partitions of $x$, is the minimal superadditive extension of $f$.

Proof. We will show that $F$ is superadditive. The minimality of $F$ will then follow from the fact that any superadditive extension $\hat{f}$ of $f$ must satisfy $\hat{f}(x) \geqq \Sigma f\left(x^{i}\right)$ for all $x \in E$ and all $a$-partitions $x^{1}, \cdots, x^{n}$ of $x$. Let $x, y \in E, \varepsilon>0$. Choose $a$-partitions $x^{1}, \cdots, x^{m}$ and $y^{1}, \cdots, y^{n}$ for

Received November 6, 1959. This paper is part of the author's doctoral thesis, and the author is indebted to Professor John Green for his guidance in its preparation. Thanks are also due the National Science Foundation for their support. 
$x$ and $y$ respectively such that $f\left(x^{1}\right)+\cdots+f\left(x^{m}\right) \geqq F(x)-\varepsilon / 2$ and $f\left(y^{1}\right)+\cdots+f\left(y^{n}\right) \geqq F(y)-\varepsilon / 2$. Then the numbers $x^{1}, \cdots, x^{n}, y^{1}, \cdots, y^{n}$ form an $a$-partition for $x+y$ and we have

$$
\begin{aligned}
F(x+y) \geqq f\left(x^{1}\right)+\cdots & +f\left(x^{m}\right)+f\left(y^{1}\right)+\cdots+f\left(y^{n}\right) \\
& \geqq F(x)+F(y)-\varepsilon .
\end{aligned}
$$

Suppose we have an approximation for $F(x)$ : that is, a number $\varepsilon>0$ and an $a$-partition $x^{1}, \cdots, x^{n}$ for $x$ such that $F(x)-\Sigma f\left(x^{i}\right)<\varepsilon$. If among the members of this $a$-partition there are two, say $x^{j}$ and $x^{k}$ such that $u=x^{j}+x^{k} \leqq a$, then since $f(u) \geqq f\left(x^{j}\right)+f\left(x^{k}\right)$, we have

$$
F(x)-\left[f(u)+\sum_{i \neq j, k} f\left(x^{i}\right)\right] \leqq F(x)-\sum_{1}^{n} f\left(x^{i}\right)<\varepsilon .
$$

In other words, replacing two numbers used in the approximation by their sum $u \leqq a$ yields an approximation at least as good as the original. It follows that if $x$ satisfies the inequality $(M-2) a / 2 \leqq x \leqq(M-1) a / 2$, where $M$ is a positive integer, then there exist arbitrarily good approximations for $F(x)$ using only $M$ terms in the $a$-partition. If $f$ is continuous, then a simple compactness argument results in the following theorem:

THEOREM 2. Let $f$ be a continuous superadditive function on [0, a], and let $F$ be its minimal superadditive extension. Let $x$ satisfy the inequality $(M-2) a / 2 \leqq x \leqq(M-1) a / 2$. Then $\exists$ an a-partition $x^{1}, \cdots, x^{M}$ for $x$ such that

$$
\Sigma f\left(x^{i}\right)=F(x) .
$$

Such an $a$-partition for $x$ will be called a decomposition of $x$, for which we shall use the notation $\langle x\rangle$ whenever convenient. We will denote by $N(x)$ a number so large that for any continuous superadditive function on $[0, a], \exists$ a decomposition $\langle x\rangle$ of $x$ with at most $N(x)$ members. It follows from the above that we can always let $N(x)=2 x / a+2$, for example.

Henceforth we shall be concerned primarily with continuous nonnegative superadditive functions for which we shall use the abbreviation $c s a$. It is readily verified that such functions are non-decreasing and vanish at the origin.

2. Combinations of extensions. One might expect that if the members of a family $f$ of csa functions are combined in a linear fashion to give another csa function $h$, then combining the members of the family $\tilde{f}$ of minimal superadditive extensions of functions in $f$ in the same way would give rise to a function $H$ which is the minimal superadditive 
extension of $h$. However this is not always the case. Consider, for example, the functions $f$ and $g$ defined on [0,3] as follows: $f(0)=0$, $f(1)=0, f(2)=0, f(3)=1$ and $g(0)=0, g(1)=0, g(2)=2, g(3)=3, f$ and $g$ linear on $[n, n+1], n=0,1,2$. Simple computations show that whereas $(F+G)(4)=5$ and $F G(4)=4$, the minimal superadditive extensions of $f+g$ and $f g$ take on the values 4 and 3 respectively at $x=4$. The minimal superadditive extension of a sum (product) of superadditive functions is thus not necessarily the sum (product) of the minimal superadditive extensions. However, some processes do commute with taking minimal superadditive extensions.

THEOREM 3. Let $\left\{f_{n}\right\}$ be a sequence of csa functions converging to the continuous function $f$ on $I=[0, a]$. Let $f_{n}{ }^{*} F_{n}$. Then $f$ is csa and $f^{*} \lim _{n \rightarrow \infty} F_{n}$.

Proof. That $f$ is superadditive and non-negative is clear. Since for each positive integer $n$ the function $f_{n}$ is non-decreasing, the convergence of $\left\{f_{n}\right\}$ to $f$ is uniform on $I$. Given $\varepsilon>0$ and $x \in E$, let $M$ be such that $n \geqq M \Rightarrow \max _{t \in I}\left|f_{n}(t)-f(t)\right|<\varepsilon / N(x)$ where $N(x)$ is a number chosen as in $\S 1$. Let $k>M$ and let $\left\langle x^{k}\right\rangle \equiv x_{k}^{1}, \cdots, x_{k}^{N^{(x)}}$ and $\langle x\rangle \equiv x^{1}$, $\cdots, x^{N(x)}$ be decompositions for $x$ relative to $F_{k}$ and $F$ respectively. We have

$$
F(x)=\sum_{i=1}^{N(x)} f\left(x^{i}\right) \geqq \sum_{i=1}^{N(x)} f\left(x_{k}^{i}\right)
$$

and

$$
F_{k}(x)=\sum_{i=1}^{N(x)} f_{k}\left(x_{k}^{i}\right) \geqq \sum_{i=1}^{N(x)} f_{k}\left(x^{i}\right) .
$$

It follows from these two inequalities that

$$
F(x)-F_{k}(x) \mid<\varepsilon,
$$

for $n \geqq M$.

3. Behavior of the minimal superadditive extension. It seems reasonable to expect that the minimal superadditive extension $F$ of a $\operatorname{cs} \alpha$ function $f$ will enjoy many of the properties of $f$. To a certain extent this is true and we are able to predict much about the behavior of $F$ by examining the behavior of $f$.

THEOREM 4. Let $f$ be csa on $[0, a]$. If $f^{*} F$, then $F$ is csa on $E$.

Proof. Clearly $F$ is non-negative. To prove that $F$ is continuous let $\varepsilon>0$ and choose $\delta<a / 2 \ni$ if $u, v \leqq a$ and $|u-v|<\delta$ then $|f(u)-f(v)|<\varepsilon$. Now let $x$ and $y$ be points of $E$ for which $|y-x|<\delta$, 
say $y=x+h$. Let $\langle y\rangle=y^{1}, \cdots, y^{N}$ be a decomposition for $y$ with, say, $y^{1}, \geqq a / 2$. We have

$$
F(y)=\sum_{1}^{N} f\left(y_{i}\right) \text { and } F(x) \geqq \sum_{2}^{N} f\left(y^{i}\right)+f\left(y^{1}-h\right) .
$$

Hence $0 \leqq F(y)-F(x) \leqq f\left(y^{1}\right)-f\left(y^{1}-h\right)<\varepsilon$.

In a similar manner one can establish the following theorem, which is stated without proof.

Theorem 5. Let $f$ be csa on $[0, a]$. If $f^{*} F$, then the following statements hold:

(a) If $f$ satisfies a Lipschitz condition with coefficient $M$, then so does $F$;

(b) If $\langle y\rangle=y^{1}, \cdots, y^{M}$ is a decomposition for $y$ and $f$ is differentiable at $y^{i}$ and $y^{j}$, then $f^{\prime}\left(y^{i}\right)=f^{\prime}\left(y^{j}\right)$. If, in addition, $F$ is differentiable at $y$, then $F^{\prime}(y)=f^{\prime}\left(y^{i}\right)$.

One might expect that the differentiability of $f$ on $[0, a]$ would imply the differentiability of $F$, except possibly at integral multiples of a. Although this turns out not to be the case, we do have the following theorem:

THEOREM 6. Let $f$ be a csa function on the interval [0, a], with $f^{\prime}$ continuous on $(0, a)$. For $x$ not an integral multiple of $a$, let $X$ be the set of points of $[0, a]$ which can be used in a decomposition for $x$. Then $F$ has a right hand derivative $F_{+}(x)$ and a left hand derivative $F_{-}(x)$ at $x$ with

$$
F_{+}(x)=\sup _{t \in X} f^{\prime}(t) \equiv S
$$

and

$$
F_{-}(x)=\inf _{t \in X} f^{\prime}(t) \equiv I .
$$

Proof. We will prove only the upper equality. The lower can be proved in a similar manner. It suffices to show $D^{+} F(x)=D_{+} F(x)=S$ where $D^{+} F$ and $D_{+} F$ are the upper and lower right hand derivatives of $F$. Suppose $\exists \varepsilon>0 \ni D^{+} F(x)>S+2 \varepsilon$. Then a sequence $\left\{h_{i}\right\}$ of numbers approaching 0 such that

$$
F(x)<F\left(x+h_{i}\right)-(S+\varepsilon) h_{i}
$$

for $i=1,2, \cdots$. For each positive integer $i$, let $\left(u^{i}, v^{i}, \cdots, w^{i}\right)$ be a decomposition for $x+h_{i}$. Without loss of generality, we assume that the sequence $\left(u^{i}, v^{i}, \cdots, w^{i}\right)$ converges to, say, $(u, v, \cdots, w)$; otherwise we consider a convergent subsequence. Since $x$ is not an integral multiple of $a$, one of the numbers $u, v, \cdots, w$ is not equal to 0 or $a$. Denote such a one by $u$. From (1) we have 


$$
F(x)<f\left(u^{i}\right)+f\left(v^{i}\right)+\cdots+f\left(w^{i}\right)-(S+\varepsilon) h_{i} .
$$

Choose $N_{1} \ni i>N_{1}$ implies that

$$
f\left(u^{i}\right)<f\left(u^{i}-h_{i}\right)+\left[f^{\prime}\left(u^{i}-h_{i}\right)+\varepsilon / 2\right] h_{i} .
$$

That $N_{1}$ can be so chosen follows from the continuity of $f^{\prime}$. In fact, let $\delta$ be such that $|u-v|<\delta \Rightarrow\left|f^{\prime}(u)-f^{\prime}(v)\right|<\varepsilon / 4$. Now choose $N_{1}$ such that $i>N_{1} \Rightarrow u-\delta<u^{i}-h_{i}<u^{i}<u+\delta$. If $y \in\left[u^{i}-h_{i}, u^{i}\right]$, with $i>N_{1}$, then $f^{\prime}\left(u^{i}-h_{i}\right)+\varepsilon / 2>f^{\prime}(y)$. Hence (3) is a valid inequality. For $i>N_{1}$ we have from (2) and (3),

$$
F(x)<f\left(u^{i}-h_{i}\right)+f\left(v^{i}\right)+\cdots+f\left(w^{i}\right)+\left[f^{\prime}\left(u^{i}-h_{i}\right)-(S+\varepsilon / 2)\right] h_{i} .
$$

Now the sequence $\left(u^{i}-h_{i}, v^{i}, \cdots, w^{i}\right)$ converges to $(u, v, \cdots, w)$ and $u+v+\cdots+w=x$. Thus, since

$$
f\left(u^{i}\right)+f\left(v^{i}\right)+\cdots+f\left(w^{i}\right)=F\left(x+h_{i}\right) \geq F(x),
$$

and $F$ is a superadditive function, we have

$$
f(u)+f(v)+\cdots+f(w)=F(x)
$$

and $u \in X$. Therefore $f^{\prime}(u) \leqq S$. By the continuity of $f^{\prime}, \lim _{i \rightarrow \infty} f^{\prime}\left(u^{i}-h_{i}\right)=$ $f^{\prime}(u)$. Hence $\exists$ a positive number $N_{2}$ such that $i>N_{2} \Rightarrow f^{\prime}\left(u^{i}-h_{i}\right)<S+\varepsilon / 2$. Let $i=\max \left(N_{1}, N_{2}\right)$. For this $i$ we have from (4),

$$
F(x)<f\left(u^{i}-h_{i}\right)+f\left(v^{i}\right)+\cdots+f\left(w^{i}\right) .
$$

This is impossible, for $u^{i}-h_{i}+v^{i}+\cdots+w^{i}=x$ for each $i=1,2, \cdots$ and $F$ is superadditive. We have shown $D^{+} F(x) \leqq S$.

It remains to show $D_{+} F(x) \geqq S$. Let $\varepsilon>0$, and let $(u, v, \cdots, w)$ be a decomposition for $x$ such that $u \neq a$, and $f^{\prime}(u)>S-\varepsilon / 4$. Choose $\delta>0 \ni h<\delta \Rightarrow f(u+h)>f(u)+(S-\varepsilon / 2) h_{i}$. For $h<\delta$,

$$
F(x+h) \geq f(u+h)+f(v)+\cdots+f(w)>F(x)+(S-\varepsilon / 2) h .
$$

The first and third members of this inequality give

$$
\frac{F(x+h)-F(x)}{h}>S+\varepsilon / 2 .
$$

Since $\varepsilon$ was arbitrary, $D_{+} F(x) \geqq S$, and the proof of the theorem is complete.

We now proceed to obtain a linear upper bound for $F$. If $f$ is csa on $[0, a]$, then the function $g$ defined by $g^{\prime}(x)=f(x) / x$ is continuous on $[0, a]$ and satisfies $g(n x) \geqq g(x), n=1,2, \cdots$, whenever $n x \leqq a$. It follows that $g$ attains a maximum at some point of $(0, a]$.

Theorem 7. Let $f$ be csa on $[0, a], f^{*} F$, and let $g$ be defined as 
above. Let $t$ be a point of $(0, a]$ at which $g$ attains its maximum $M$. Then

(a) $F(x) / x \leq M$ for all $x>0$,

(b) $F(x) / x=M$ if $x$ is an integral multiple of $t$,

(c) $\lim _{x \rightarrow \infty} F(x) / x=M$,

(d) $\max _{x \in[0, a]}[M x-f(x)]=\max _{x \in E}[M x-F(x)]$,

(e) $\lim _{x \rightarrow \infty}[F(x)-M x]=0$ if $f$ is differentiable at $t$.

Proof. The proofs of (a), (b), (c) and (d) are straightforward and will be omitted. Let us then turn to (e). For each $x \in E$, write $x$ in the form $x=n t+y$, where $n$ is an integer and $0 \leqq y<t$. Define a function $F^{*}$ by $F^{*}(n t+y)=n f(t+y / n), n=1,2, \cdots$. Clearly $F^{*}(x) \leqq$ $F(x) \leqq M x$ for all $x \in E$. We will show that $\lim _{x \rightarrow \infty}\left[M x-F^{*}(x)\right]=0$. By the definition of $F^{*}$ we have

$$
M x-F^{*}(x)=M(n t+y)-n f(t+y / n) .
$$

Noting that $f(t)=M t$, we see that the right hand member of this last equation can be written in the form

$$
y\left[M-\frac{f(t+y / n)-f(t)}{y / n}\right]
$$

Now let $x \rightarrow \infty$. Then $y$ is bounded between 0 and $t$ and $n \rightarrow \infty$. The expression (1) approaches 0 , since $f^{\prime}(t)=M$.

We observe that the function $F^{*}$ of the preceding theorem is asymptotic to $F$ with $F^{*} \leqq F$. Hence $F(x)$ is bounded between $F^{*}(x)$ and $M x$, two functions which are easy to calculate, and whose difference is small when $x$ is large.

4. The polygonal method. The minimal superadditive extension of a csa function may also be obtained as the limit of a sequence of polygonal functions. A function $p$ is said to be polygonal if $p$ is continuous and piecewise linear. The point $x \in[0, a]$ is called a vertex of $p$ if $(x$, $p(x))$ is a vertex of the polygon forming the graph of $p$.

THEOREM 8. Let $p$ be polygonal on $[0, a]$ with equally spaced vertices. Then $p$ is superadditive if and only if $p$ is superadditive on its vertices.

Proof. If $p$ is superadditive, then $p$ is clearly superadditive on its vertices. To prove the converse consider the function $g$ defined on the set

$$
D \equiv\{(x, y): 0 \leqq x, y \leqq \alpha \text { and } x+y \leqq a\}
$$


by the equation $g(x, y)=p(x+y)-p(x)-p(y)$. It is easy to verify that $g$ is planar on any triangle $T$ of the form

$$
T=\left\{(x, y): u_{1} \leqq x \leqq u_{2} ; v_{1} \leqq y \leqq v_{2}, x+y \leqq(\text { or } \geqq) u_{2}+v_{2}\right\},
$$

where $\left(u_{1}, v_{1}\right)$ and $\left(u_{2}, v_{2}\right)$ are pairs of successive vertices of $p$. Hence $g$ attains its minimum on $T$ at one of the points $\left(u_{i}, v_{i}\right)$ and therefore its minimum on $D$ at a point $(u, v)$ where both $u$ and $v$ are vertices of $p$. Thus, if $g$ is anywhere negative then $g$ is negative at a point whose two coordinates are vertices of $p$. This proves the theorem.

Now let $p$ be a polygonal function on $[0, a]$ with vertices at $0, v, 2 v$, $\cdots, m v=a$. We define a function $P$ on $E$ as follows:

$$
\begin{aligned}
P(x) & =p(x) \quad \text { for } x \leqq a \\
P(M v) & =\max _{K=1, \cdots, M-1}[P(k v)+P(M v-k v)] M \text { an integer } \geqq m+1
\end{aligned}
$$

and

$P$ linear on $[M v,(M+1) v], M=m, m+1, \cdots$.

$P$ will be called the function associated with $p$. It is easy to see that if $p$ is csa, then $P$ is csa.

Definition. A sequence $\left\{p_{n}\right\}$ of functions defined on $[0, a]$ is called a $p$-sequence if

(i) each $p_{n}$ is a polygonal function

(ii) the vertices of $p_{n}$ are $K a / 2^{n}$,

$$
K=0,1, \cdots, 2^{n}
$$

(iii) $P_{n}\left(K a / 2^{m}\right)=p_{m}\left(K a / 2^{m}\right)$ if $m \leqq n$.

In terms of this concept we have

THEOREM 9. Let $\left\{p_{n}\right\}$ be a p-sequence coverging to the csa function $f$ on $[0, a]$. For each positive integer $n$ let $P_{n}$ be the function associated with $p_{n}$. Then, if $f^{*} F,\left\{P_{n}\right\}$ converges to $F$ on $E$.

Proof. It suffices to show that $P_{n}$ approaches $F$ on $[0,2 a]$. Let $F^{*}(x)=\varlimsup_{\lim _{n \rightarrow \infty}} P_{n}(x)$. It is easy to check that $F^{*}$ is superadditive. Let $V_{k}$ be the set of vertices of $P_{k}$ in $[a, 2 \alpha]$, and let $V=\bigcup_{1}^{\infty} V_{k}$. If $v \in V$, then $\lim _{n \rightarrow \infty} P_{n}(v)$ exists since the sequence $\left\{P_{n}(v)\right\}$ is ultimately non-decreasing and $P_{n}(v) \leqq F(v)$ for all $n$. We have $\lim _{n \rightarrow \infty} P_{n}(v) \leqq F(v)$. But since $F^{*}$ is superadditive, we have $F^{*} \geqq F$. Hence $F^{*}=F$ on $V$. By standard arguments involving the continuity of $F$, the density of $V$ in $[a, 2 a]$, and the monotonicity of each $P_{n}$ and $F^{*}$, it follows that $F \equiv$ $F^{*}$ and that $F^{*}=\lim _{n \rightarrow \infty} P_{n}(x)$.

5. Superadditive functions in $n$-dimensions. It turns out that many of the results obtained in one dimension have their analogues in $n$-di- 
mensions. The interval $I \equiv[0, a]$ is replaced by a fundamental region $R$ defined by the inequalities $0 \leqq x_{i} \leqq a_{i}, i=1, \cdots, n$, where the $a_{i}$ are arbitrary positive numbers. The decomposition method works, just as it does on the line, and we can prove with little difficulty that to any superadditive function $f$ on $R$ there corresponds a minimal superadditive extension $F$ to $E_{n}^{+} \equiv\left\{\left(x_{1}, \cdots, x_{n}\right): 0 \leqq x_{i}, i=1, \cdots, \cdots, n\right\}$. We can also prove a theorem corresponding to Theorem 5 , the derivatives here being directional derivatives. In Theorem 7 a certain line $l(x)=M x$ played an important role. In $n$-dimensions, for each direction $\theta$ we have a plane $P$, which plays the role of $l$ in some direction, and when the function $P$, defined on the fundamental region $R$ by the equation

$$
P(z)=\inf _{\theta} P_{\theta}(z),
$$

is extended to $E_{n}^{+}$by homogeneity it is the minimal concave superadditive function which bounds $F$ from above. It can be proved, at least in $E_{2}^{+}$, that

$$
n \max _{z \in R}[P(z)-f(z)] \geqq \max _{z \in E_{h}^{+}}[P(z)-F(z)] .
$$

\section{BIBLIOGRAPHY}

1. E. Hille and R. Phillips, Functional analysis and semi-groups, Amer. Math. Soc. Colloquim Publications vol. XXXI ch. 7 pp. 237-255.

2. R. A. Rosenbaum, Subadditive functions, Duke Math J., 17 (1950), p 227-247.

UNIVERSITY OF CALIFORNIA,

SANTA BARBARA 